\title{
INTRODUCTION TO THE SPECIAL ISSUE ON ROLE OF SCALABLE COMPUTING AND DATA ANALYTICS IN EVOLUTION OF INTERNET OF THINGS
}

\author{
J.N. SWAMINATHAN* GOPI RAM ${ }^{\dagger}$ AND SUREKA LANKA
}

The evolution of Internet of Things has given way to a Smart World where there is an improved integration of devices, systems and processes in humans through all pervasive connectivity. Anytime, anywhere connection and transaction is the motto of the Internet of things which brings comfort to the users and sweeps the problem of physical boundary out of the way. Once it has come into the purview of developers, new areas have been identified and new applications have been introduced. Small wearables which can track your health to big automated vehicles which can move from one place to another self navigating without human intervention are the order of the day. This has also brought into existence a new technology called cloud, since with IoT comes a large number of devices connected to the internet continuously pumping data into the cloud for storage and processing. Another area benefited from the evolution of IoT is the wireless and wired connectivity through a wide range of connectivity standards.

As with any technology, it has also created a lot of concerns regarding the security, privacy and ethics. Data protection issues created by new technologies are a threat which has been recognized by developers, public and also the governing body long back. The complexity of the system arises because of the various sensors and technologies which clearly tell the pattern of the activities of the individual as well an organization making us threat prone. Moreover, the volume of the data in the cloud makes it too difficult to recognize the privacy requirement of the data or to segregate open data from private data. Data analytics is another technology which supposedly increases the opportunity of increasing business by studying this private data collected from IoT and exploring ways to monetize them. It also helps the individual by recognizing their priorities and narrowing their search. But the data collected are real world data and aggregation of this data in the cloud is an open invitation to the hackers to study about the behaviors of the individuals.

The special issues of Scalable Computing has attract related to the Role of Scalable Computing and Data Analytics in Evolution of Internet of Things has attracted 28 submissions from which were selected 12. In what follows we present them shortly:

1. Murali et. al has executed on detecting brain tumor using thresholding and histogram techniques.

2. Suresh et. al has introduced an ensemble integrated security system with cross breed technique.

3. Shahidha Bhanu has done background modeling using a Q-Tree based foreground segmentation.

4. Agalya et. al has executed topological ordering signal selection techniques for iot based devices. The work has been funded under dst-inspire program.

5. Karthikeyan et. al has introduced secured identity based cryptosystem approach for intelligent routing protocol in vanet.

6. Indragandhi et.al has developed an application based ETLP scheme on heterogeneous multicore embedded system for real time image processing.

7. Putta Venkata Subbaiah et.al has done a study to analyze enhancement techniques on sound quality for bone conduction and air conduction speech processing.

8. Kuppusamy et.al has introduced a novel approach based on modified cycle generative adversarial networks for image steganography.

\footnotetext{
${ }^{*}$ QIS College of Engineering and Technology, Ongole, Andhra Pradesh, India

${ }^{\dagger}$ National Institute of Technology, Warangal, Telangana, India

${ }^{\ddagger}$ Stamford International University, Bangkok, Thailand.
} 
9. Jairam Naik et.al has executed minimizing deadline misses and total run-time with load balancing for a connected car systems in fog computing.

10. Antony Joseph Rajan et. al has proposed long and strong security using reputation and ecc for cloud assisted wireless sensor networks.

11. Pavithra et. al has detected and classified 2D and 3D hyper spectral image using enhanced harris corner detector.

12. Rengarajan et. al has proposed secured privacy-conserving authentication scheme using cuckoo filter in vanet.

13. Hema et. al has introduced logistics optimization in supply chain management using clustering algorithms.

14. Jayanthi et. al has executed acoustic feedback cancellation in efficient hearing aids using genetic algorithm.

15. Kusma et. al has analysed on deep learning methods for ECG based cardiovascular disease prediction.

16. Finally Rajalakshmi et. al has introduced a hybrid intrusion detection system for mobile adhoc networks using fbid protocol.

Acknowledgement: The Guest editorial members want to acknowledge Dr. N. S. Kalyan Chakravarthy, Chairman \& Correspondent, QIS Group of Institutions, Ongole, Andhra Pradesh. 